\title{
Addendum zur Laudatio Univ.-Prof. Dr. Eberhard Gabriel zum 80. Geburtstag
}

\author{
Georg Psota
}

Online publiziert: 19. Mai 2020

(C) Springer-Verlag GmbH Austria, ein Teil von Springer Nature 2020

Die folgenden Zeilen resultieren einerseits daraus, dass bei der Laudatio für Eberhard Gabriel die Nennung einer für die Entwicklung der Psychiatrie in Wien maßgeblichen Persönlichkeit unterblieben und daher nachzuholen ist. Konkret handelt es sich um den für die Wiener Psychiatriereform verantwortlichen Politiker und Mediziner Univ.-Prof. Dr. Alois Stacher. Er war als Stadtrat für Gesundheit und Soziales zweifelsohne eine starke und tatkräftige Stütze für den 1978 zum ärztlichen Direktor ernannten Eberhard Gabriel und gab ihm auch den politischen Rückhalt für weitreichende Spitalsreformen.

Zum anderen bezieht sich dieses Addendum auch auf eine weitere Erkenntnis des Psychiatrie-Historikers Eberhard Gabriel, der wiederholt darauf aufmerksam macht, dass Reformen vor allem dann gelingen, wenn es zu einem guten Zusammenspiel von Professionisten und Politikern kommt. Zweifelsohne gilt das auch für die Psychiatrie. Ein medizinhistorischer Aspekt, der nicht zu vernachlässigen ist, und es im Gegenteil verdient, immer wieder aufs neue ausreichend berücksichtigt und beleuchtet zu werden.

Interessenkonflikt G. Psota gibt an, dass kein Interessenkonflikt besteht.

Hinweis des Verlags Der Verlag bleibt in Hinblick auf geografische Zuordnungen und Gebietsbezeichnungen in veröffentlichten Karten und Institutsadressen neutral.

Die Laudatio Hartmann Hinterhuber, Theodor Meißel, Georg Psota, Hans Schanda. Univ.-Prof. Dr. Eberhard Gabriel zum 80. Geburtstag finden Sie unter Neuropsychiatr 2020;34 (2): https://doi.org/10.1007/s40211-020-00342-0.

\section{Dr. G. Psota $(\bowtie)$}

Kuratorium für Psychosoziale Dienste in Wien,

Modecenterstraße 14/A/2. OG, 1030 Wien, Österreich

chapost@psd-wien.at 